\title{
Efficacy Analysis of Percutaneous Endoscopic Spinal Surgery for Young Patients with Discogenic Low Back Pain
}

\author{
Jianan Zhang $\mathbb{D}^{\prime}$, Qichang Li', Yu Du', Zhengjian Yan', Liang Chen², Liyuan Wang' \\ 'Department of Orthopedics, The Second Affiliated Hospital of Chongqing Medical University, Chongqing, 4000I0, People's Republic of China; \\ ${ }^{2}$ Department of Musculoskeletal Tumor, Chongqing University Cancer Hospital \& Chongqing Cancer Institute \& Chongqing Cancer Hospital, \\ Chongqing, 400030, People's Republic of China
}

Correspondence: Liyuan Wang, Department of Orthopedics, The Second Affiliated Hospital of Chongqing Medical University, Chongqing, 4000I0, People's Republic of China, Tel +89-13648380803, Email wanglynana@I63.com

Purpose: To evaluate the application value of percutaneous endoscopic spinal surgery for young patients with discogenic low back pain (DLBP) and to judge its clinical efficacy.

Methods: We retrospectively analyzed young patients with single-segment discogenic lumbago from July 2018 to June 2020 in our department who underwent percutaneous endoscopic surgery according to the inclusion and exclusion criteria. We finally enrolled 20 patients. The follow-up time was 6-30 months. In all patients, we recorded the visual analog scale (VAS) score for waist pain and the Oswestry Disability Index (ODI) preoperatively, immediately postoperatively and at the last follow-up. We used the modified MacNab criteria to assess the curative effect at the last follow-up.

Results: All 20 patients underwent successful operations without complications. No recurrence was observed during follow-up. The VAS score of low back pain was $5.05 \pm 1.19$ points before surgery, $1.50 \pm 051$ points immediately after surgery, and $1.10 \pm 0.72$ points at the last follow-up ( $\mathrm{P}<0.05$ preoperative vs both postoperative). At the last follow-up, the VAS scores of all 20 patients were $\leq 2$, and 4 patients had no pain. The ODI was $46.66 \pm 7.03 \%$ before surgery, $9.78 \pm 4.05 \%$ immediately after surgery, and $4.11 \pm 3.18 \%$ at the last $(\mathrm{P}$ $<0.05$, preoperative vs both postoperative). According to the evaluation under the modified MacNab standard, the good-excellent rate of clinical efficacy at the last follow-up was $95 \%$.

Conclusion: Percutaneous endoscopic spinal surgery can significantly improve the symptoms and dysfunction of young patients with DLBP and has little effect on the biomechanical stability of the lumbar spine. This surgery has great clinical application value.

Keywords: percutaneous endoscopic spinal surgery, discogenic low back pain, young patients, transforaminal, interlaminar

\section{Key Message}

At present, there are many controversies about the treatment of discogenic low back pain. This study retrospectively analyzed the efficacy of percutaneous endoscopic spinal surgery for young patients with discogenic low back pain, and the results show that this method is effective in the short and medium term.

\section{Introduction}

Discogenic low back pain (DLBP) is a kind of chronic nonspecific low back pain with a high incidence. ${ }^{1,2}$ It is mainly resting low back pain caused by structural and functional disorders of the disc due to intervertebral disc degeneration. With the increase in unhealthy living and working styles in modern times, patients are showing DLBP earlier in life. Long-term chronic pain has a great impact on quality of life, causing a great burden on the social economy and other aspects of life, and it can even cause disability. ${ }^{1-3}$

In essence, DLBP is caused by a series of pathological changes caused by disc degeneration. Due to the long-term bad posture of the young patients, the intervertebral disc showed a series of degenerative reactions, such as dehydration of the 
nucleus pulposus and "black", the formation of annulus fissure, the formation of pannus mediated by inflammatory factors, and the entry of nerve endings into annulus fibrosus with inflammatory granulation tissue. When a large amount of water is lost in the nucleus pulposus, components such as hyaluronic acid, keratinized sulfate, glycoprotein and collagen fibers also change accordingly, leading to changes such as intervertebral disc thinning, structural destruction, height reduction and annulus fibrosus rupture. ${ }^{3}$ As the annulus ruptures, the autoimmune system recognizes and produces inflammatory mediators. ${ }^{4-6}$ Induction of inflammatory mediators promotes the formation of local pannus and granulation tissue and stimulates nerve fiber endings to enter the annulus fibrosus, leading to low back pain. ${ }^{7,8}$

There is much debate about the diagnosis and treatment of DLBP, and the current treatment strategies are mainly divided into two types: nonsurgical treatment and surgical treatment. ${ }^{2,3,9}$ Nonsurgical treatment includes drug therapy, functional exercise, massage and acupuncture, but they often have a poor therapeutic effect. Some patients need to take nonsteroidal anti-inflammatory drugs for a long time to control their symptoms, and some patients even need opioids, but gastrointestinal discomfort and other drug complications can easily occur. Surgical treatments include lumbar fusion and radiofrequency thermocoagulation, electrothermal therapy, and intradiscal methylene blue injection. The shortcomings of these treatments include uncertain efficacy and many complications, and there is still no standard, effective treatment for DLBP. $^{2,10-13}$ Especially for young patients, while effectively alleviating the symptoms of low back pain, it is often necessary to be more careful in the selection of the treatment method to preserve the physiological function of the lumbar spine.

With the continuous advancement of percutaneous endoscopic surgical technology, which has the advantages of less trauma, high safety, high effectiveness, high visibility, and less influence on spinal stability, these techniques have been widely applied in the treatment of lumbar diseases, with good clinical efficacy. ${ }^{14,15}$ In this study, percutaneous endoscopic spinal surgery was used to treat young patients with DLBP, and its clinical efficacy was observed to evaluate the application value of this surgery for DLBP.

\section{Methods}

\section{Inclusion Criteria and Exclusion Criteria}

Inclusion criteria: (1) history of chronic low back pain for $\geq 1$ year with ineffective standard nonsurgical treatment for $\geq 3$ months; (2) disc degeneration and a high-intensity zone (HIZ) on MRI (Figure 1); (3) single-segment lesions; (4) no serious underlying diseases; (5) treatment with percutaneous endoscopic spinal surgery; (6) age between 20 and 50.
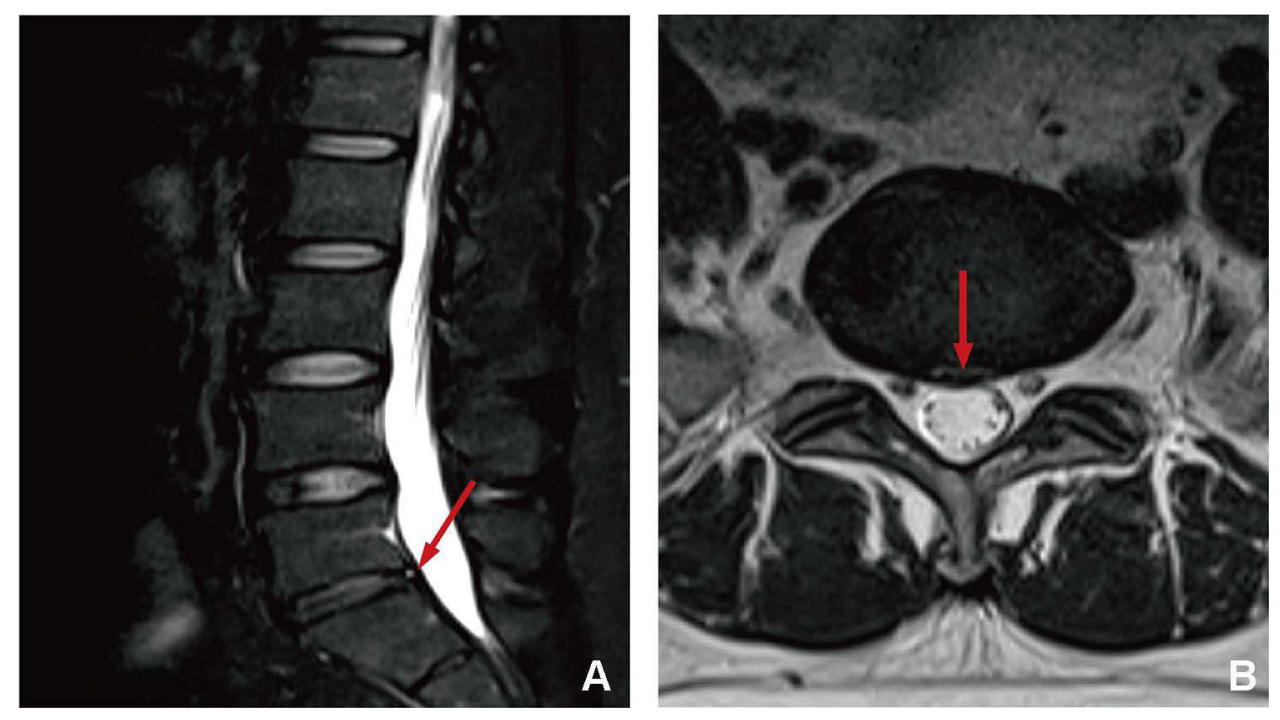

Figure I Preoperative lumbar MRI T2-weighted image showing mild degenerative changes in the lumbar spine. The red arrow indicates the HIZ, which is annular fissure. (A) sagittal plane. (B) coronal plane. 
The exclusion criteria were as follows: (1) low back pain complicated with lumbar spinal stenosis, lumbar instability or spondylolisthesis; (2) pathological changes in the lumbar spine, such as compression fractures, infections, or tumors; (3) previous history of spinal surgery; and (4) moderate to severe anxiety, depression or other mental disorders or cognitive dysfunction.

\section{General Information}

This study was approved by the medical research ethics committee of our hospital (ethics review document number: Research Ethics Review No. 40, 2021). According to the inclusion and exclusion criteria, 20 young patients with singlelevel DLBP treated by percutaneous endoscopic spinal surgery in our department from July 2018 to June 2020 were enrolled. They were 12 males and 8 females, aged 37.35 \pm 8.16 years. Seven patients had L4/5 DLBP and 13 patients L5/ S1. Follow-up time was 6-30 months (Table 1).

\section{Operative Technique}

Surgical approach: Different surgical approaches were selected according to the affected segment. An intervertebral foraminal approach was used for the L4/5 segment, and an interlaminar approach was used for the L5/S1 segment.

\section{Transforaminal Approach}

The patient was treated with intravenous anesthesia combined with local anesthesia. First, the patient lay prone on the arch of the operating table (Figure 2A), and local disinfection was applied. After the HIZ position was confirmed on preoperative MRI, X-ray C-arm fluoroscopy was used to locate the L4/5 intervertebral foramen, and $10 \mathrm{~cm}$ from the spinous process was set as the puncture point (Figure 2B). After local anesthesia with lidocaine diluent (1:1) at the fixed point, oblique puncture was performed with a puncture needle to the L4/5 intervertebral foramen. No blood was withdrawn from the syringe. Five more milliliters of lidocaine diluent was injected when the needle had been withdrawn to the fascia, and $2 \mathrm{~mL}$ lidocaine diluent was injected when the needle had been withdrawn back to the subcutaneous region. After the anesthesia was completed, a positioning needle punctured the puncture point and then slowly inserted under C-arm fluoroscopy. Anteroposterior film showed that the puncture needle was located outside the L4/5 intervertebral space (Figure 2C), and lateral film showed that the puncture needle was located at the lower edge of the L4/5 intervertebral foramen (Figure 2D). The guide wire was inserted, the pencil head was replaced, the expansion tube was expanded step by step, and the working channel was placed. The channel was attached to the facet bone, and an endoscopic system was placed. After the surrounding soft tissue was removed, the superior facet of L5 was exposed, and facet arthroplasty was performed by using a ring saw (Figure 2E). The soft tissues outside the spinal canal were separated with basket forceps and nucleus pulposus forceps. Banket forceps were used to remove the ligamentum flavum and enter the spinal canal.

Endoscopy revealed that fissures had formed in the outer layer of the intervertebral disc annulus, surrounded by a small amount of inflammatory tissue and blood vessels, and the HIZ nodules were composed of a large number of pannus (Figure 3A). After sufficient prehemostasis, the HIZ nodules were removed, the nucleus pulposus tissue around the fissures was cleaned, and the surrounding inflammatory lesions and hemostasis were eliminated by radiofrequency ablation (Figure 3B and C). At the same time, the thermal effect of radiofrequency ablation was used to retract the annulus fissure opening. The cracks visible under the microscope mostly disappeared, and the annulus integrity was mostly restored. The pulsation of the walking nerve root was examined (Figure 3D). After no obvious protrusion was

Table I Patients' Demographic Characteristics

\begin{tabular}{|l|c|c|c|c|}
\hline \multicolumn{2}{|l|}{ Gender } & \multirow{2}{*}{$\begin{array}{c}\text { Age (Years) } \\
\text { Male }\end{array}$} & \multicolumn{2}{|c|}{ Lesion Level } \\
\cline { 5 - 5 } & Female & & L4/5 & L5/S I \\
\hline 12 & 8 & $37.35 \pm 8.16$ & 7 & 13 \\
\hline
\end{tabular}



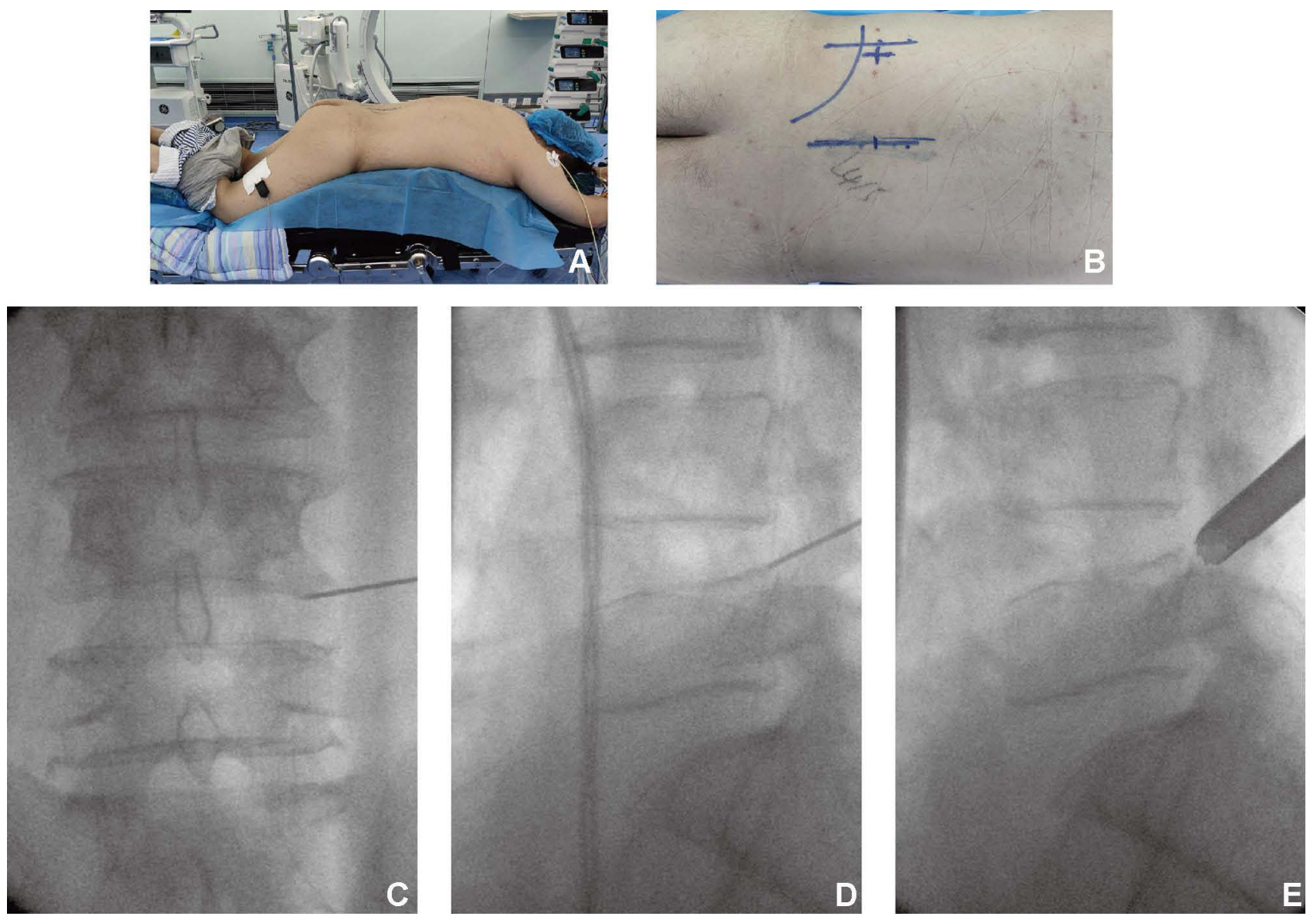

Figure 2 Transforaminal endoscopic spinal surgery. (A) The patient lies prone on the arched frame of the operating table after anesthesia. (B) The Location of puncture point of foraminal approach. (C) On the C-arm fluoroscopic anteroposterior radiograph, the puncture needle was located lateral to the L4/5 intervertebral space. (D) Lateral radiograph shows the puncture needle located at the lower edge of the L4/5 foramen. (E) Using ring saw to shape facet at L5 superior articular process.

detected, radiofrequency ablation was used again to stop the bleeding. After full hemostasis, the endoscope system was removed, and the incision was sutured.

\section{Interlaminar Approach}

The patient was placed prone on the arch of the operating table under general anesthesia. C-arm fluoroscopy was used to locate the intervertebral space of L5 and S1 after disinfecting and laying the tissue. The puncture point was located at the projection point of the body surface $2 \mathrm{~cm}$ from the posterior midline of the intervertebral space (Figure 4A). A puncture needle was used to puncture the L5/S1 lamina under repeat C-arm fluoroscopy. After the puncture was successful, the guide needle was inserted, the dilating tube was expanded step by step (Figure 4B), and the working channel and endoscopic system were placed.

Endoscopic operation: Radiofrequency ablation was used under endoscopy to completely remove HIZ and surrounding inflammatory tissue. The annulus fissure opening was retracted under the thermal effect of radiofrequency ablation, which restored the integrity of annulus fibrosus. After confirming that there was no nucleus pulposus tissue in the spinal canal, sufficient hemostasis was performed on the operative area. After that the endoscope was removed and the incision was sutured.

\section{Follow-Up}

Patients underwent lumbar MRI examination within 1 day after surgery to evaluate the decompression effect. They got out of bed wearing waist circumference support 2-3 days after surgery. All patients were followed up with outpatient and telephone consultations. A visual analog scale (VAS) was used to assess changes in the level of low back pain, the Oswestry Disability Index (ODI) was used to assess improvements in lumbar function, and a modified MacNab standard 

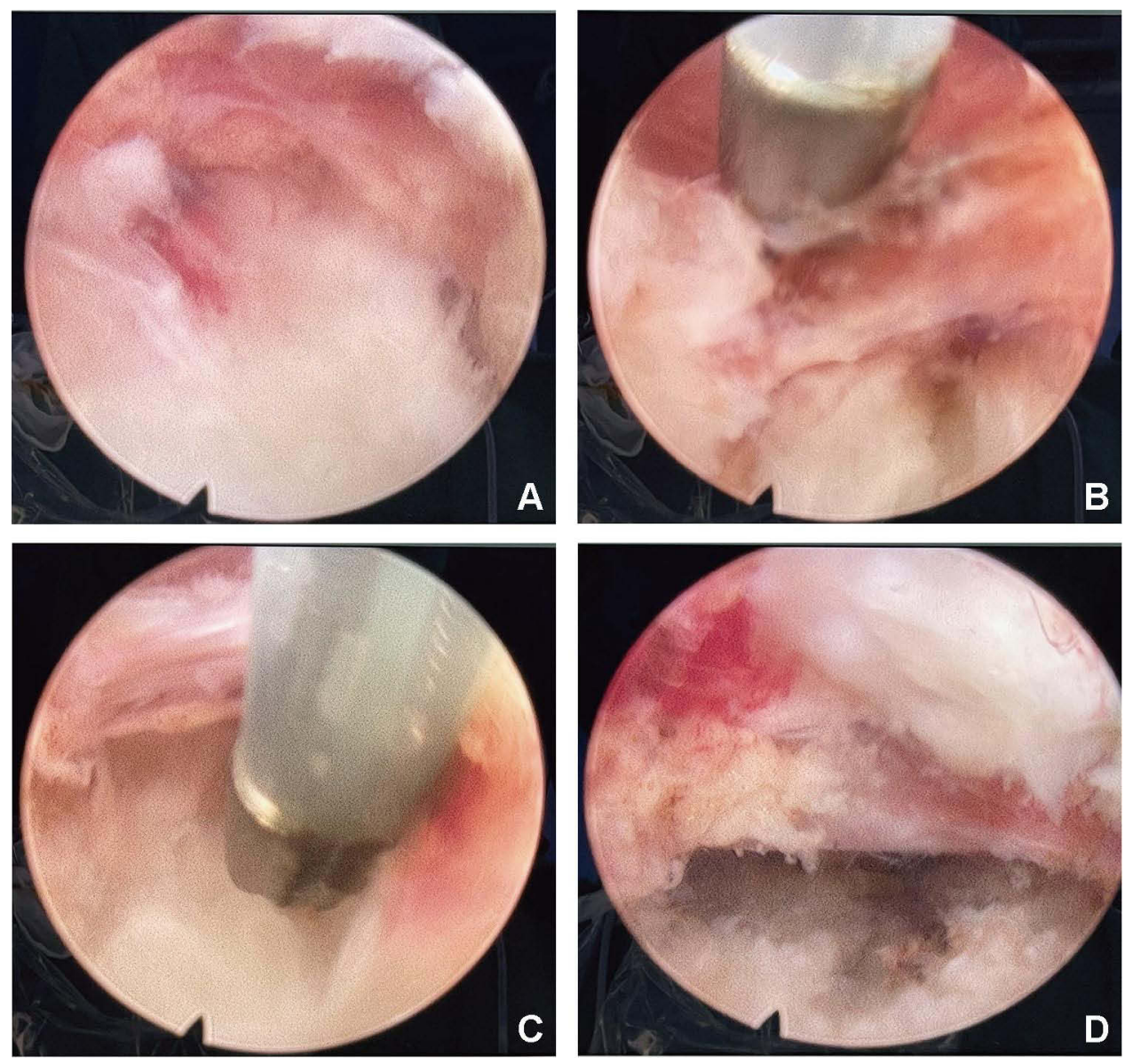

Figure 3 Percutaneous spinal endoscopic procedure. (A) Endoscopy showed hyperplasia of pannus, hyperemia and edema, and ventral annular fibrous dome-like projection. (B) After electric coagulation of pannus, the space between annulus fibrosus and nerve root was dissociated. (C) Dissociate up and down along the nerve root to separate the adhesive tissue and expose the decompression space. (D) The nerve root collapsed and ventral compression was relieved after removal of the ruptured annulus fibrosus.

was used to assess clinical outcomes at the last follow-up. Lumbar dynamic radiographs were taken at the last follow-up to evaluate stability. Lumbar MRI was also performed in 12 patients who were followed up for more than 12 months, and the status of their intervertebral discs was assessed using the modified Pfirrmann scale.

\section{Statistical Methods}

SPSS 23.0 software was used for statistical analysis. VAS score and ODI are expressed as $\mathrm{x} \pm \mathrm{s}$. The VAS score and ODI were compared at different time points before and after surgery by repeated-measures analysis of variance. Probability values less than $0.05(\mathrm{P}<0.05)$ were considered statistically significant.

\section{Results}

\section{VAS}

The operation was successful and complication-free in all 20 patients. No recurrence was observed during the follow-up period. The VAS score was $5.05 \pm 1.19$ points before surgery, $1.50 \pm 0.51$ points immediately after surgery, and $1.10 \pm 0.72$ points at the last follow-up ( $\mathrm{P}<0.05$, each postoperative time point vs preoperative) (Figure 5A). At the last follow-up, the VAS scores of all 20 patients were $\leq 2$, and 4 patients had no pain.

\section{ODI}

The ODI of the 20 patients was $46.66 \pm 7.03 \%$ before surgery, $9.78 \pm 4.05 \%$ immediately after surgery, and $4.11 \pm 3.18 \%$ at the last follow-up ( $\mathrm{P}<0.05$, each postoperative time point vs preoperative) (Figure 5B). 

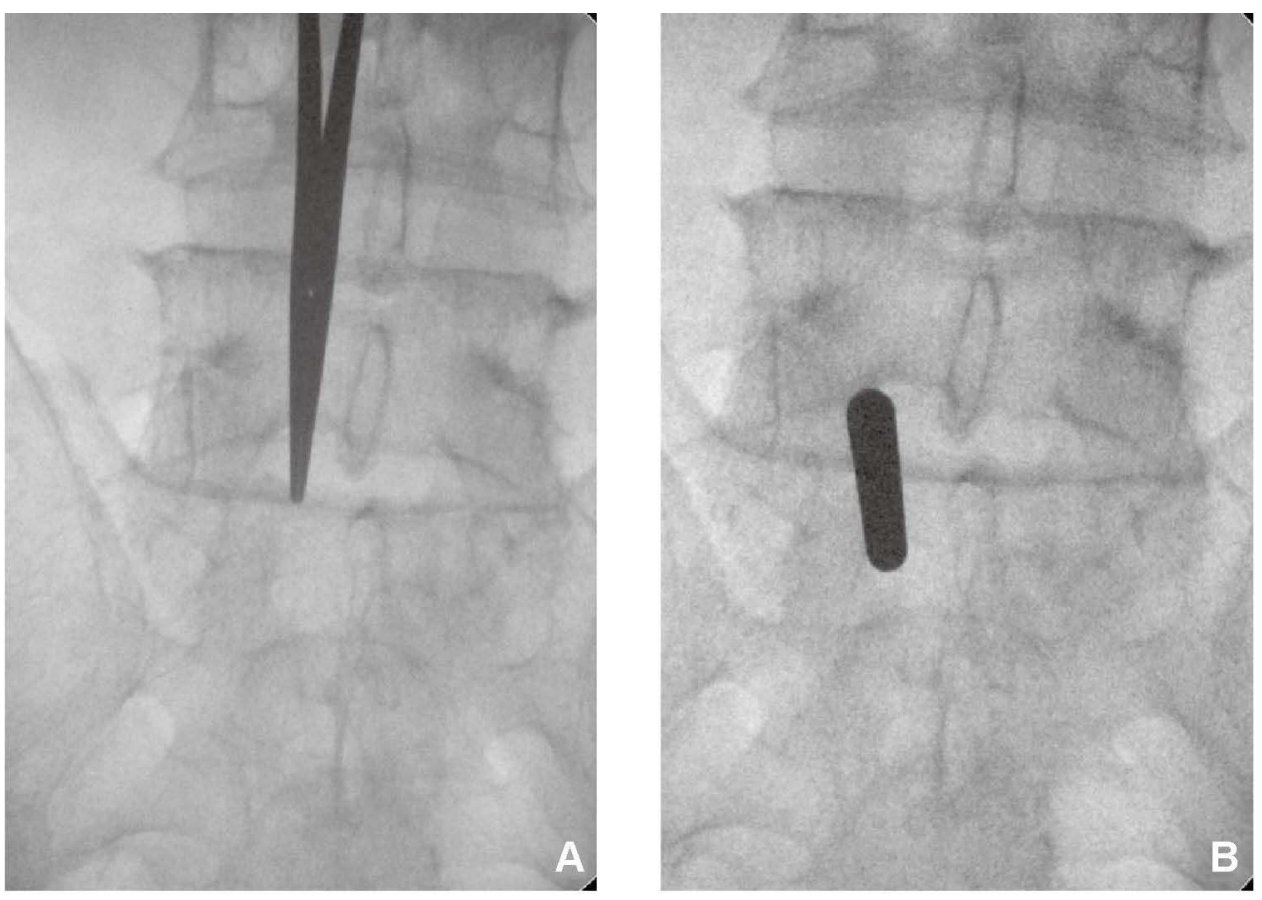

Figure 4 Interlaminar endoscopic spinal surgery. (A) The location of puncture point of interlaminar approach. (B) The L5/SI interlaminar approach was progressively expanded with dilated tube.
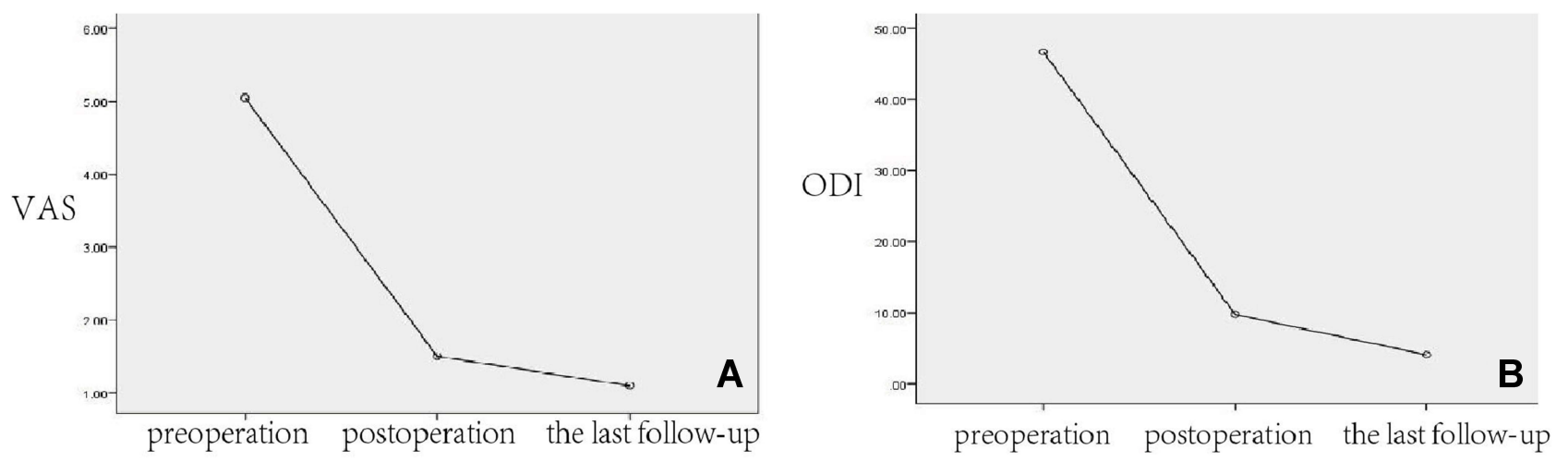

Figure $\mathbf{5}$ Changes of VAS score and ODI index of low back pain at preoperative, immediately after surgery and at the last follow-up. (A) VAS score. (B) ODI index.

\section{Modified MacNab Standard}

At the last follow-up, by evaluation under the modified MacNab standard, the clinical efficacy rate (good to excellent) and improvement rate (excellent, good or fair) of the 20 patients were $95 \%$ and $100 \%$, respectively (Figure 6 ).

\section{Imaging Examination}

Postoperative lumbar MRI showed no HIZ nodules in any patient (Figure 7). Lumbar MRI and the modified Pfirrmann grade at the last follow-up in the patients with follow-up times of more than 1 year still showed no significant worsening from the immediate postoperative period. No sign of lumbar instability was found on dynamic radiographs at the last follow-up.

\section{Discussion}

The diagnosis and treatment of DLBP has always been challenging, and its diagnosis often requires considering the patient's history, clinical symptoms, signs, imaging findings, and even serological indicators. ${ }^{1}$ After excluding other 


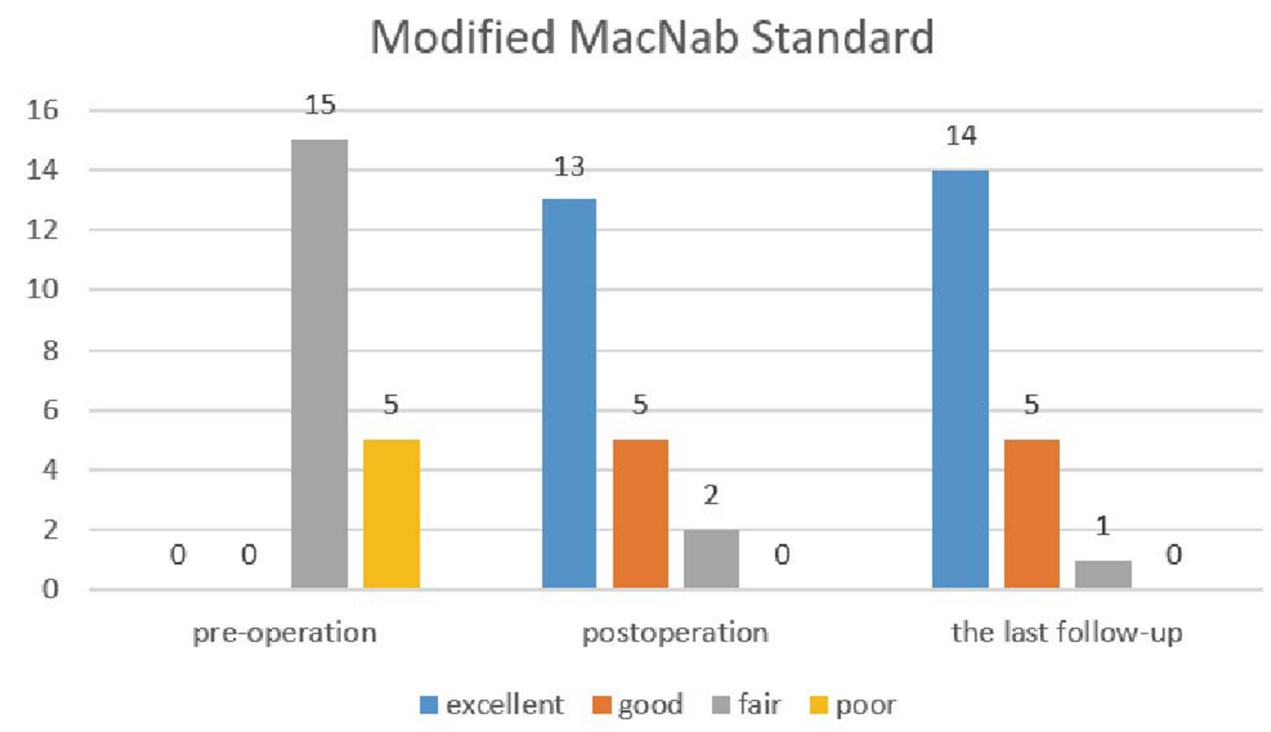

Figure 6 Clinical follow-up results according to the modified MacNab standard.
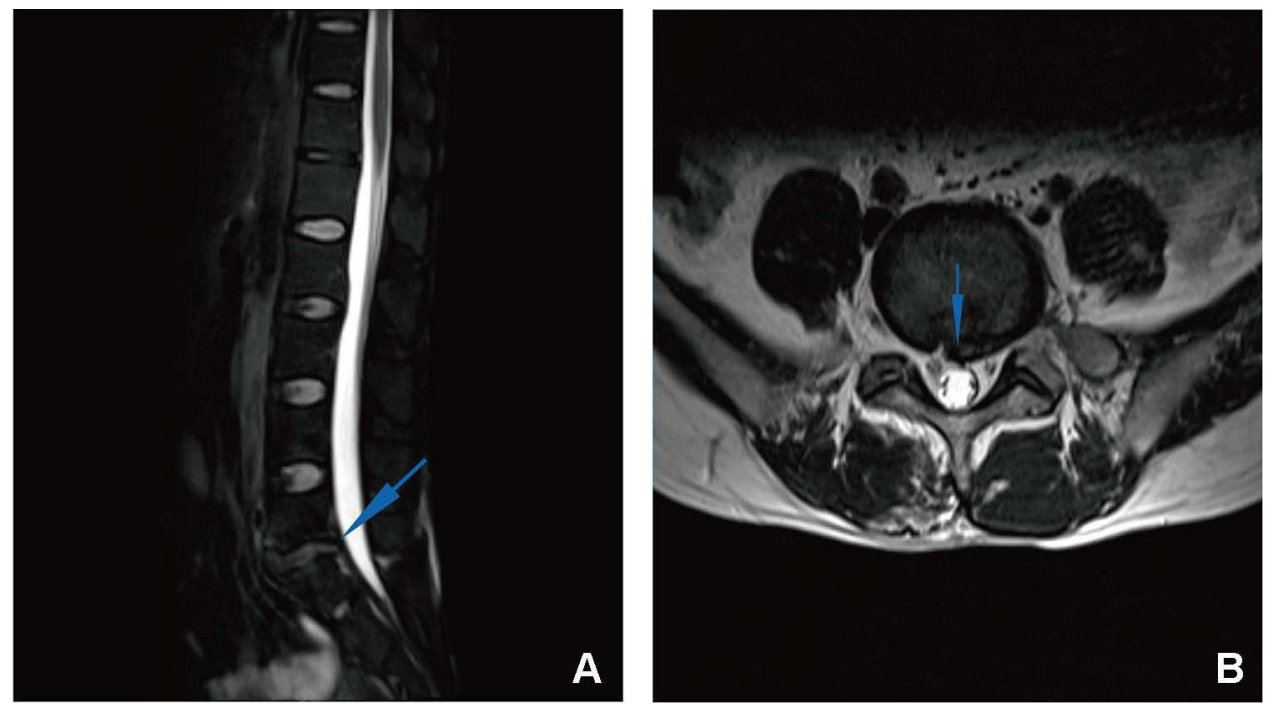

Figure 7 After the operation, the HIZ nodules were fully decompressed, the ruptured annulus fibers were removed, and the nerve roots were mildly hyperemia and edema and it's compression was disappeared. The blue arrow indicates the disappearance of HIZ after surgery. (A) sagittal plane. (B) coronal plane.

diseases causing low back pain, DLBP can be diagnosed. Clinically, DLBP is the most common type of chronic nonspecific low back pain, and its course is usually greater than 12 weeks. It is mainly characterized by recurrent resting low back pain manifesting as aggravation of pain after sitting and standing for a long time and poor relief of pain during bed rest. It may be accompanied by axial symptoms of the lower limbs. The HIZ plays an important role in the diagnosis of DLBP. The HIZ is a high-intensity region located at the posterior margin of the intervertebral disc in T2weighted lumbar MR images separated from the nucleus pulposus signal. Liu et al defined the HIZ as a lesion with a signal brightness of at least $50 \%$ of the CSF intensity. ${ }^{16}$ This is often seen as a characteristic image of DLBP. Aprill et al showed in 1992 that the HIZ was strongly associated with discogenic congruent pain with high sensitivity (71\%) and high specificity (89\%), ${ }^{17}$ which was sufficient to demonstrate that the presence of an HIZ was a marker of annulus fibrosus rupture and causing symptoms. Although subsequent studies have shown that the HIZ can also be observed on MRI in 6-31\% of normal lumbar patients with no symptoms of low back pain, ${ }^{18-20}$ Bogduk emphasized that the "lowintensity areas" found in asymptomatic people only represent the formation of annular clefts, and only when activated by 
the local inflammatory response. ${ }^{21}$ These clefts may present a high signal and cause symptoms of lower back pain. Through endoscopy during the operation, we clearly observed that the HIZ was composed of many panni. When the HIZ was touched preoperatively, consistent pain was obviously felt. After clearing the HIZ and all inflammatory lesions nearby, the symptoms of low back pain basically disappeared, strongly indicating that the stimulation of a local inflammatory reaction was the cause of the low back pain symptoms. The occurrence of an HIZ is closely related to the presence of panni and inflammatory granulation tissue. Therefore, this study suggests that the pathogenesis of DLBP is closely related to HIZ nodules.

Discography is generally considered the gold standard for the diagnosis of DLBP, but its necessity is controversial. Zhang et al mentioned that the main shortcoming of discography as a diagnostic means is that the judgment of the discography results is closely related to the pain felt by patients, but pain depends on subjective judgment, and a high false positive rate will inevitably occur among patients with psychological fear or hypersensitivity to pain. ${ }^{1}$ Discography has a high false positive rate, reaching as high as $50 \% .{ }^{1,22}$ In addition, Carragee et al showed that even the use of smalldiameter needles and limited injection pressure for disc puncture during discography may be associated with accelerated disc degeneration, disc herniation, decreased disc height, and changes in endplate signals. ${ }^{23}$ Madan et al found that discography did not significantly improve DLBP after surgical treatment. ${ }^{24}$ Therefore, discography was not applied to patients in this study to prevent complications caused by invasive intervertebral disc manipulation and to reduce the impact on intervertebral discs in these young patients, to ensure a more objective evaluation of the efficacy results of this study.

During the follow-up period of this study, the patients' low back pain symptoms significantly improved, and the VAS score and ODI were significantly reduced. At the last follow-up, the VAS score of all 20 patients was $\leq 2$, the ODI was $\leq 8.9 \%$, the good-excellent clinical efficacy rate was $95 \%$, and the improvement rate was $100 \%$. Thus, we achieved relatively satisfactory clinical efficacy. We believe that percutaneous spinal endoscopic surgery has a good symptomrelieving effect in young patients with DLBP and restores their lumbar function. At the last follow-up, no lumbar instability was found on the dynamic radiographs of any patient, and lumbar MRI showed no accelerated degeneration of the intervertebral disc, indicating that percutaneous endoscopic spinal surgery had little influence on the biomechanical stability of the lumbar spine. Percutaneous endoscopic spinal surgery for young patients with DLBP not only has a good curative effect but also does not significantly impair the lesioned segment or the structure or stability of the adjacent vertebrae, ensuring a good prognosis for young patients.

Young people have heavy learning and working burdens in modern society, and they engage in much exercise and other activity, which places higher requirements on their lumbar function. Therefore, we should pay more attention to the preservation of function while alleviating the symptoms of young patients with DLBP. In recent years, a variety of surgical methods have been introduced for the treatment of discogenic low back pain, the more popular of which include minimally invasive procedures such as radiofrequency ablation and intradiscal methylene blue injection. However, a number of studies have proposed that intradiscal methylene blue injection has no definite effect on the relief of low back pain or only maintains a good effect for a short time (6 months). ${ }^{112}$ Zhang et al also mentioned that lumbar fusion can be chosen for patients in whom conservative treatment and intradiscal methylene blue injection fail. ${ }^{11}$ However, lumbar fusion has a high risk of numerous complications that may lead to long-term and irreversible effects on patients' lumbar function, ${ }^{13}$ so it is difficult for us to recommend lumbar fusion as the treatment for young patients with DLBP. At this time, the use of percutaneous endoscopic spinal surgery for the treatment of DLBP is undoubtedly a more appropriate choice.

Tsou et al first used percutaneous endoscopic surgery to treat DLBP. ${ }^{25}$ Compared to minimally invasive treatments such as electrothermal therapy and simple radiofrequency ablation, this surgical method has the advantages of more accurate removal of the nucleus pulposus tissue embedded in the annulus fibrosus rupture and elimination of inflammatory lesions under direct vision. However, the Yeung endoscopic spine system (YESS) surgical method requires intraand intervertebral disc operation, which will inevitably cause iatrogenic injury to the annular fibrous rupture and even the intervertebral disc tissue, which may aggravate the intra- and intervertebral disc inflammatory response and destroy its biomechanical stability. Therefore, on the basis of that operation method, we accessed the intervertebral foramen or channel between vertebral plates and performed endoscopy in the spinal canal and dorsal fiber ring. Under endoscopic 
direct vision, we processed the fiber ring fracture around the mouth and back side of the vertebra near the inflammatory lesion, removed the incarcerated nucleus pulposus tissue near the crevasse, and at the same time used radiofrequency ablation to minimize scar formation in the fiber ring crevasse, This ensured the safety of visual manipulation while avoiding tearing the annulus fibrosus or affecting the internal structure of the disc and maintaining the integrity of the disc tissue. When the annulus fissure receives the thermal effect of radiofrequency ablation, the inflammatory lesions are cleared, and the nerve endings that enter the annulus with the inflammatory granulation tissue are also destroyed. During the follow-up of this study, all patients showed significant relief of the symptoms of low back pain, without recurrence, and no HIZ was observed on lumbar MRI, showing that the integrity of the intervertebral disc had been repaired and no inflammatory lesions recurred after their complete removal. At the last follow-up, the lumbar MRI of patients with a follow-up period of more than 1 year was evaluated using the modified Pfirrmann grade to evaluate the status of their intervertebral discs. Compared with their immediate postoperative intervertebral discs, no signs of accelerated degeneration were observed, showing that percutaneous endoscopic spinal surgery had little effect on intervertebral discs.

\section{Conclusion}

Percutaneous endoscopic spinal surgery has a definite curative effect on young patients with DLBP. This treatment program has many advantages, such as less trauma, strong visibility, good prognosis, and small impact on the structure of the lumbar spine, giving it great clinical application value. Percutaneous endoscopic spinal surgery may play an important role in the treatment of young patients with DLBP.

\section{Research Ethics and Consent}

We designed this study after the approval of the ethics committee of the Second Affiliated Hospital of Chongqing Medical University. (ethics review document number: Research Ethics Review No. 40, 2021). Due to COVID-19 and their work, patients were unable to return to the hospital to sign informed consent. Therefore, we only obtained verbal informed consent from the patients. The study proposal was reviewed by ethics committee of the Second Affiliated Hospital of Chongqing Medical University, and the need for written informed consent had been waived. All authors make a commitment to patient data confidentiality and compliance with the declaration of Helsinki.

\section{Funding}

This study was supported by grants from Chongqing Young and Middle-aged Medical High-end Talent Studio(No. ZQNYXGDRCGZS2018003), Smart Medicine Project of Chongqing Medical University(No.ZHYX2019020).

\section{Disclosure}

The authors declare that they have no competing interests.

\section{References}

1. Zhang Y-G, Guo T-M, Guo X, Wu S-X. Clinical diagnosis for discogenic low back pain. Int J Biol Sci. 2009;647-658. doi:10.7150/ijbs.5.647

2. Kallewaard JW, Terheggen MAMB, Groen GJ, et al. 15. Discogenic low back pain. Pain Pract. 2010;10(6):560-579. doi:10.1111/j.15332500.2010.00408.x

3. Zhao L, Manchikanti L, Kaye AD, Abd-Elsayed A. Treatment of discogenic low back pain: current treatment strategies and future options-a literature review. Curr Pain Headache Rep. 2019;23(11). doi:10.1007/s11916-019-0821-x

4. Molinos M, Almeida CR, Caldeira J, Cunha C, Goncalves RM, Barbosa MA. Inflammation in intervertebral disc degeneration and regeneration. J R Soc Interface. 2015;12(104):20141191. doi:10.1098/rsif.2014.1191

5. Kang JD, Georgescu HI, McIntyre-Larkin L, Stefanovic-Racic M, Donaldson WF 3rd, Evans CH. Herniated lumbar intervertebral discs spontaneously produce matrix metalloproteinases, nitric oxide, interleukin-6, and prostaglandin E2. Spine (Phila Pa 1976). 1996;21(3):271-277. doi:10.1097/00007632-199602010-00003

6. Peng BG. Pathophysiology, diagnosis, and treatment of discogenic low back pain. World J Orthop. 2013;4(2):42-52. doi:10.5312/wjo.v4.i2.42

7. Yang G, Liao W, Shen M, Mei H. Insight into neural mechanisms underlying discogenic back pain. $J$ Int Med Res. 2018;46(11):4427-4436. doi:10.1177/0300060518799902

8. Garcia-Cosamalon J, del Valle ME, Calavia MG, et al. Intervertebral disc, sensory nerves and neurotrophins: who is who in discogenic pain? $J$ Anat. 2010;217(1):1-15. doi:10.1111/j.1469-7580.2010.01227.x

9. Peng B, Zhang Y, Hou S, Wu W, Fu X. Intradiscal methylene blue injection for the treatment of chronic discogenic low back pain. Eur Spine J. 2007;16(1):33-38. doi:10.1007/s00586-006-0076-1 
10. Urrútia G, Kovacs F, Nishishinya MB, Olabe J. Percutaneous thermocoagulation intradiscal techniques for discogenic low back pain. Spine. 2007;32(10):1146-1154. doi:10.1097/01.brs.0000261492.55121.93

11. Zhang X, Hao J, Hu Z, Yang H. Clinical evaluation and magnetic resonance imaging assessment of intradiscal methylene blue injection for the treatment of discogenic low back pain. Pain Physician. 2016;19(8):E1189-E1195.

12. Kallewaard JW, Wintraecken VM, Geurts JW, et al. A multicenter randomized controlled trial on the efficacy of intradiscal methylene blue injection for chronic discogenic low back pain. Pain. 2019;160(4):945-953. doi:10.1097/j.pain.0000000000001475

13. Tay BBK, Berven S. Indications, techniques, and complications of lumbar interbody fusion. Semin Neurol. 2002;22(2):221-230. doi:10.1055/ S-2002-36545

14. Mayer HM. A history of endoscopic lumbar spine surgery: what have we learnt? Biomed Res Int. 2019;2019:1-8. doi:10.1155/2019/4583943

15. Chen Q, Zhang Z, Liu B, Liu S. Evaluation of percutaneous transforaminal endoscopic discectomy in the treatment of lumbar disc herniation: a retrospective study. Orthop Surg. 2021;13(2):599-607.

16. Liu C, Cai H-X, Zhang J-F, Ma -J-J, Lu Y-J, Fan S-W. Quantitative estimation of the high-intensity zone in the lumbar spine: comparison between the symptomatic and asymptomatic population. Spine J. 2014;14(3):391-396. doi:10.1016/j.spinee.2013.06.078

17. Aprill C, Bogduk N. High-intensity zone: a diagnostic sign of painful lumbar disc on magnetic resonance imaging. Br J Radiol. 1992;65 (773):361-369. doi:10.1259/0007-1285-65-773-361

18. Carragee EJ, Paragioudakis SJ, Khurana S. 2000 Volvo Award winner in clinical studies: lumbar high-intensity zone and discography in subjects without low back problems. Spine (Phila Pa 1976). 2000;25(23):2987-2992. doi:10.1097/00007632-200012010-00005

19. Weishaupt D, Zanetti M, Hodler J, Boos N. MR imaging of the lumbar spine: prevalence of intervertebral disk extrusion and sequestration, nerve root compression, end plate abnormalities, and osteoarthritis of the facet joints in asymptomatic volunteers. Radiology. 1998;209(3):661-666. doi:10.1148/radiology.209.3.9844656

20. Stadnik TW, Lee RR, Coen HL, Neirynck EC, Buisseret TS, Osteaux MJ. Annular tears and disk herniation: prevalence and contrast enhancement on MR images in the absence of low back pain or sciatica. Radiology. 1998;206(1):49-55. doi:10.1148/radiology.206.1.9423651

21. Bogduk N. Point of view: predictive signs of discogenic lumbar pain on magnetic resonance imaging with discography correlation. Spine. 1998;23 (11):1259-1260. doi:10.1097/00007632-199806010-00017

22. Carragee EJ, Tanner CM, Yang B, Brito JL, Truong T. False-positive findings on lumbar discography. Spine. 1999;24(23):2542. doi:10.1097/ 00007632-199912010-00017

23. Carragee EJ, Don AS, Hurwitz EL, Cuellar JM, Carrino J, Herzog R. 2009 ISSLS Prize winner: does discography cause accelerated progression of degeneration changes in the lumbar disc. Spine. 2009;34(21):2338-2345. doi:10.1097/BRS.0b013e3181ab5432

24. Madan S, Gundanna M, Harley JM, Boeree NR, Sampson M. Does provocative discography screening of discogenic back pain improve surgical outcome? J Spinal Disord Tech. 2002;15(3):245-251. doi:10.1097/00024720-200206000-00014

25. Tsou PM, Alan Yeung C, Yeung AT. Posterolateral transforaminal selective endoscopic discectomy and thermal annuloplasty for chronic lumbar discogenic pain: a minimal access visualized intradiscal surgical procedure. Spine J. 2004;4(5):564-573. doi:10.1016/j.spinee.2004.01.014

Journal of Pain Research

Dovepress

\section{Publish your work in this journal}

The Journal of Pain Research is an international, peer reviewed, open access, online journal that welcomes laboratory and clinical findings in the fields of pain research and the prevention and management of pain. Original research, reviews, symposium reports, hypothesis formation and commentaries are all considered for publication. The manuscript management system is completely online and includes a very quick and fair peer-review system, which is all easy to use. Visit http://www.dovepress.com/testimonials.php to read real quotes from published authors.

Submit your manuscript here: https://www.dovepress.com/journal-of-pain-research-journal 